\title{
REDUCING THE IMPACT OF ELECTROCONDUCTIVITY AND THE GAP BETWEEN THE PIPE AND THE TRANSDUCER AT MEASURING THICKNESS OF ELECTROCONDUCTIVE PIPE WALLS USING THE EDDY-CURRENT METHOD
}

\author{
Evgeny Yakimov ${ }^{*}$ and Daniil Ustyugov \\ Tomsk Polytechnic University, Institute of Non-Destructive Testing, 634050, Tomsk, Russia
}

\begin{abstract}
The paper describes a dual-frequency method for reducing the impact of changes in the gap size between the eddy-current transducer and the pipe, as well as the pipe electrical conductivity on the eddy-current thickness gauge readings. A block-diagram of the dual-frequency eddycurrent thickness gauge is proposed for light-alloy drill pipes. The amplitude and signal phase dependencies on the wall thickness in the range from 6 to $17 \mathrm{~mm}$ and the gap in the range from 0 to $13.5 \mathrm{~mm}$ were studied, the results are presented. The digital signal processing algorithms based on the piecewise-linear approximation of low-frequency and high-frequency signal phase dependencies on the wall thickness are proposed. It is shown that the proposed correction algorithms can reduce the error caused by variations of electrical conductivity and the gap between the transducer and the pipe.
\end{abstract}

\section{Introduction. Measuring the thickness of pipe walls}

Light-alloy drill pipes of TB and TBP types made of aluminum alloy D16 are manufactured in accordance with the Russian standard GOST 23786-79 [1]. The pipes are used to perform the workover and the structure, exploration and production drilling. The pipe walls are worn during in-service use, and therefore the wall thickness of the main pipe section must be controlled according to the Russian standard. With due regard to the assortment, the nominal wall thickness of the main pipe section can vary from 7.5 to $13 \mathrm{~mm}$. The limit thickness deviation shall not exceed $\pm(0.4-0.5) \mathrm{mm}$ for pipes of high accuracy and $\pm(0.7-1.7) \mathrm{mm}$ for pipes of normal accuracy. A non-destructive testing method is not considered. The measuring instruments must have the accuracy of $\pm 0.1 \mathrm{~mm}$.

\footnotetext{
Corresponding author: shishkovka@mail.ru
} 
Ultrasonic devices provide high accuracy of measurement, and therefore are used to measure the thickness of metal objects. However, a good acoustic contact (through the liquid layer) is required, that decreases the speed of testing.

The advantages of the eddy-current testing method are high-performance control and sensitivity, and the possibility of non-contact measurement. These advantages enable the method automation and provide a continuous control along the entire length of the pipe. However, signals of the eddy-current transducer considerably depend on the material electrical conductivity and the gap between the transducer and the pipe.

In the previous paper [2], it was shown that a dual-frequency eddy-current method can reduce the dependence of thickness gauge readings on the gap size. Consider the fact that the method is capable of reducing the impact of changes in the pipe electrical conductivity on the thickness measurement results. With this, the block diagram of the gauge (Figure 1) comprising the surface eddy-current transducer (ECT), generator (G), amplitude and phase detectors (APD), data acquisition board with ADC, and personal computer (PC) can be used.

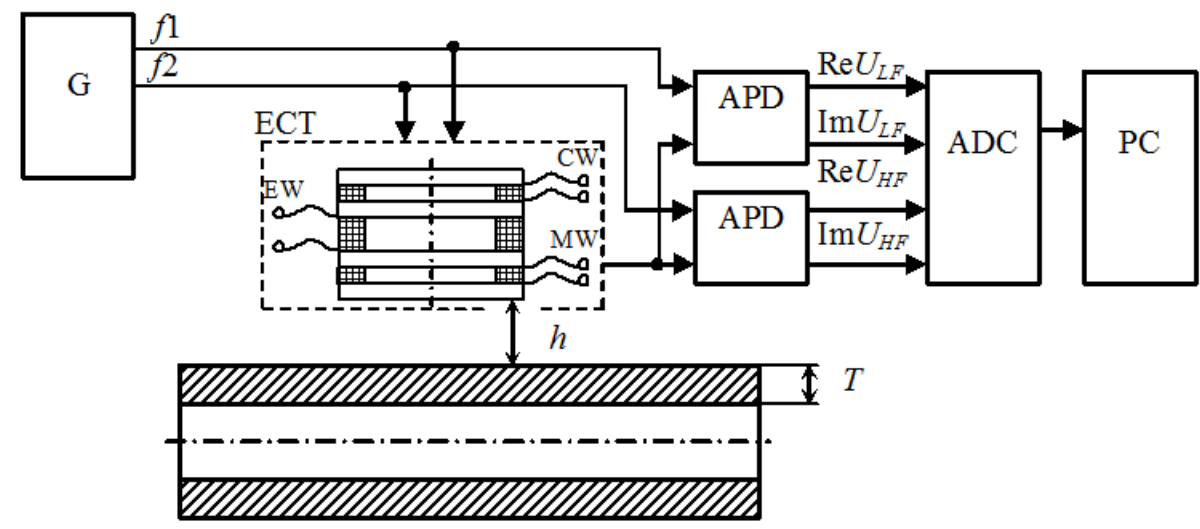

Figure 1. Block diagram of the thickness gauge based on the dual-frequency eddy-current method.

The eddy-current transducer comprises the excitation winding (EW), measurement winding (MW) and compensation winding (CW). The signal is fed from the generator to the transducer EW, which is the sum of two harmonic signals. The first frequency $f 1$ is principal and is intended to measure the wall thickness $T$. The frequency must be up to several hundred hertz and therefore is referred to as low frequency (LF). The second frequency $f 2$ is selected so that the signals of this frequency slightly depend on the pipe wall thickness and at the same time change with variations of the pipe electrical conductivity and the gap $h$ between the pipe and the transducer. These properties appear in the frequency of several kilohertz, so the frequency is called high frequency (HF).

During the measurement, signals from the eddy-current transducer arrive at the amplitude and phase detectors, which output voltages are proportional to the amplitudes of imaginary and real components of the low-frequency signal $f 1$ and high-frequency signal f2: $\operatorname{Re} U_{L F}, \operatorname{Im} U_{L F}, \operatorname{Re} U_{H F}, \operatorname{Im} U_{H F}$.

Then, signals arrive at the data acquisition board with ADC, which performs analogdigital conversion of signals and transfer them to the PC.

The PC records and processes the data.

The values of the ECT signals are used to calculate the amplitude $A_{H F}$ and phase $\varphi_{H F}$ of the high-frequency signal $(f 2)$, and the phase $\varphi_{L F}$ of the low-frequency signal $(f 1)$ : 


$$
\begin{aligned}
& A_{H F}=\sqrt{\left(\operatorname{Re} U_{H F}\right)^{2}+\left(\operatorname{Im} U_{H F}\right)^{2}}, \\
& \varphi_{H F}=\operatorname{arctg}\left(\operatorname{Im} U_{H F} / \operatorname{Re} U_{H F}\right), \\
& \varphi_{L F}=\operatorname{arctg}\left(\operatorname{Im} U_{L F} / \operatorname{Re} U_{L F}\right) .
\end{aligned}
$$

The value of the high-frequency signal amplitude $A_{H F}$ is taken to calculate the gap $h$ between the ECT and the pipe surface:

$$
h=K h \cdot\left[c g_{0}+c g_{1} \cdot e^{c g_{2} \cdot a m p}+c g_{3} \cdot e^{c g_{4} \cdot a m p}\right],
$$

where $c g_{k}$ is constant coefficients, $a m p=A_{H F} / A_{H F 0}$ is the relative amplitude of the highfrequency signal, $A_{H F 0}$ is the amplitude of the high-frequency signal for zero gap, and $K h$ is a correction factor used for non-zero gap calibration. [2].

The approximation error in accordance with expression (4) does not exceed $0.08 \mathrm{~mm}$

The dependence of the wall thickness $T$ on the low-frequency signal phase $\varphi_{L F}$ for a fixed value of the gap $h$ (Figure 2) can be approximated by the piecewise-linear function

$$
T=K_{L F}(h, j t x) \cdot\left\lfloor\varphi_{L F}+\Delta \varphi_{L F(t x)}+\Delta \varphi_{L F}+K c \cdot \varphi_{L F}\right\rfloor+B_{L F}(h, j t x),
$$

where $K_{L F}(h, j t x)$ and $B_{L F}(h, j t x)$ are the coefficients, which depend on the gap $h$ and the number $j t x$ of the site, where the thickness depends on the phase $\varphi_{L F} ; \Delta \varphi_{L F}$ and $K c$ are the coefficients used to calibrate the readings by the two known values of the pipe thickness; $\Delta \varphi_{L F(t x)}$ is the phase correction for the change in the electrical conductivity.

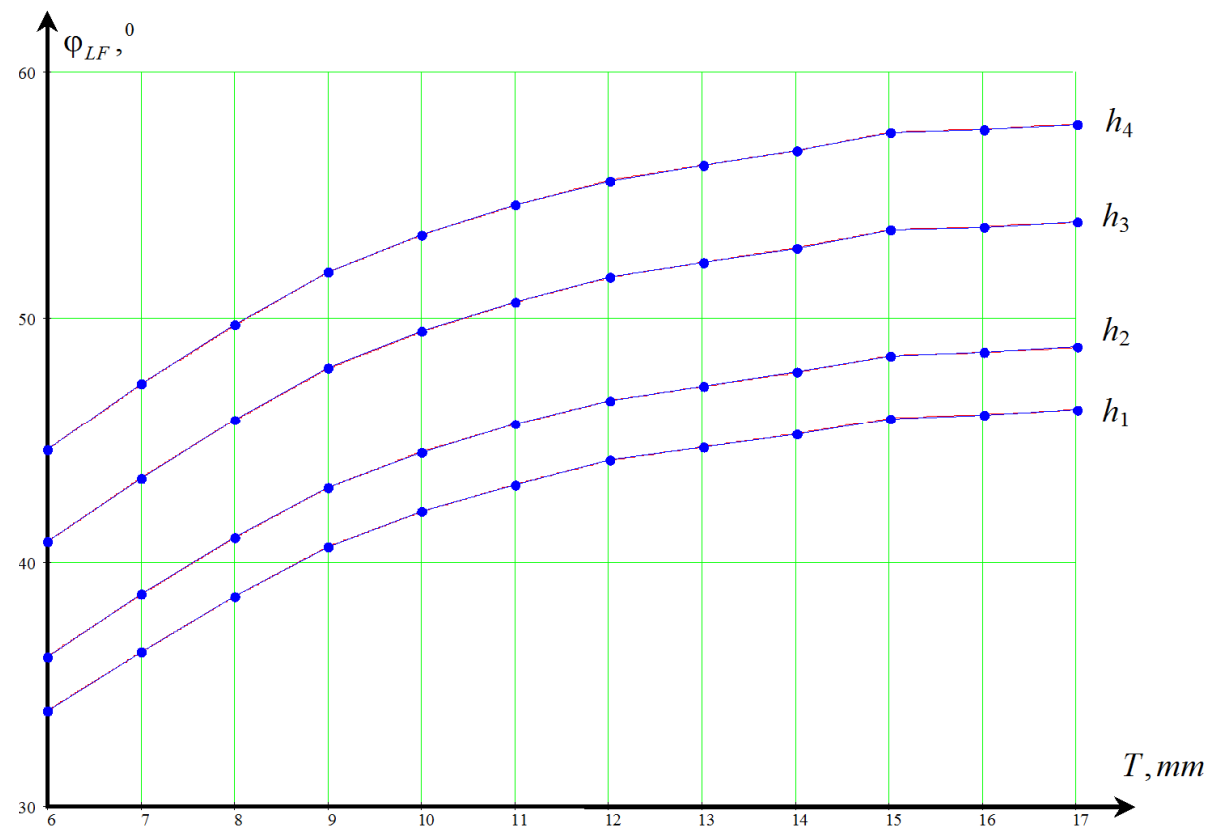

Figure 2. Dependence of the low-frequency signal phase $\varphi_{L F}$ on the pipe wall thickness $T$ for the fixed values of the gap $h$. 
The correction is calculated based on the assumption that the conductivity is temperature dependent [3-10]. However, the electrical conductivity can vary due to the different chemical composition of the tube material.

The phase dependence on the temperature can be approximated by the linear function

$$
\Delta \varphi_{L F(t x)}=-K t_{L F} \cdot(t x-t 0),
$$

where $K t_{L F}$ is the coefficient of proportionality, $t x$ is the pipe temperature, and $t 0$ is the temperature at which the thickness gauge was calibrated.

The pipe temperature is calculated from the difference of the measured high-frequency signal phase $\varphi_{H F(t x)}$ and the phase $\varphi_{H F(t 0)}$ measured at temperature $t 0$,

$$
t x=t 0-K t_{H F} \cdot\left\lfloor\varphi_{H F(t x)}-\varphi_{H F(t 0)}\right\rfloor,
$$

where $K t_{H F}$ is the coefficient of proportionality.

That is, the preliminary evaluation of the thickness is made with no regard to the conductivity correction, and then the expected value of the high-frequency signal phase $\varphi_{H F(t 0)}$ and the phase difference $\left\lfloor\varphi_{H F(t x)}-\varphi_{H F(t))}\right\rfloor$ are calculated. The change in the highfrequency signal phase against the gap $h$ and the thickness of the tube $T$ are taken into account (Figure 3). A piecewise-linear model of dependence is used

$$
\varphi_{H F(t 0)}=T \cdot\left\lfloor K k_{H F(j h x)} \cdot h+B k_{H F(j h x)}\right\rfloor+\left\lfloor K b_{H F(j h x)} \cdot h+B b_{H F(j h x)}\right\rfloor,
$$

where $K k_{H F(j h x)}, B k_{H F(j h x)}, K b_{H F(j h x)}$ and $B b_{H F(j h x)}$ are the coefficients of piecewise-linear approximation of the dependence of the high-frequency signal phase on the gap.

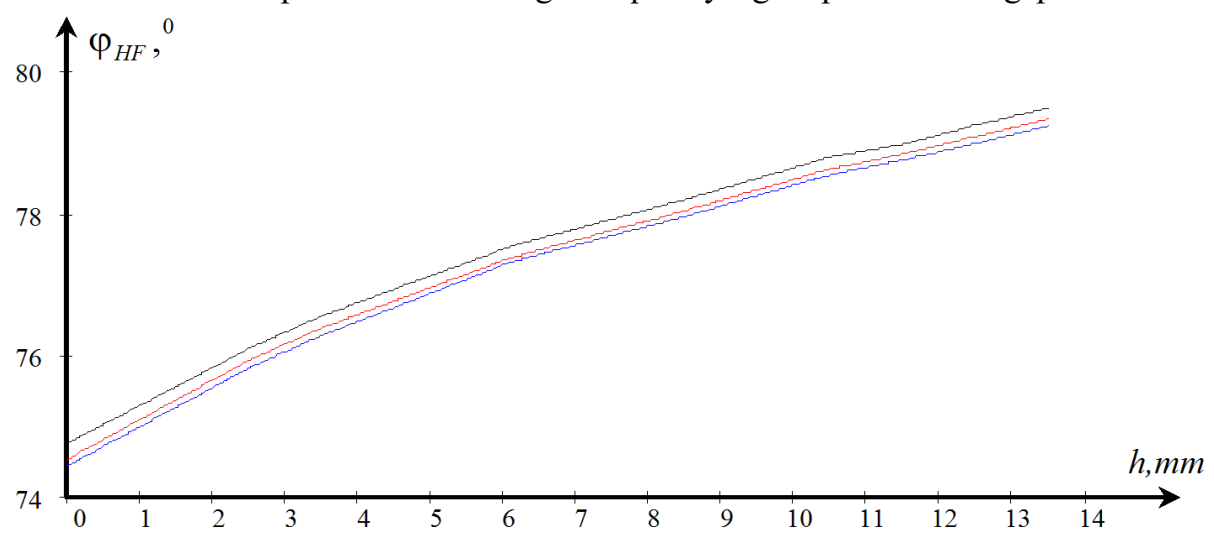

Figure 3. Dependence of the high-frequency signal phase $\varphi_{H F}$ on the gap $h$ for the fixed pipe wall thickness $T$.

\section{Thickness gauge calibration}

To calibrate the readings when measuring a zero gap, the ECT is placed directly on the pipe and the high-frequency signal amplitude $A_{H F 0}$ is saved in the memory.

For the non-zero gap calibration, the ECT is placed on the plate of known thickness and the correction factor $K h$ used in equation (4) is calculated 


$$
K h=h 2 / h .
$$

The thickness calibration is performed in two steps.

At the first step, the ECT is installed on the sample pipe with a known thickness value $T 1$, and the ECT signals are saved in the memory. Next, the ECT is installed on the sample pipe with a known value $T 2$, and the ECT signals are saved in the memory. The thickness of sample 1 should be less than the thickness of sample 2 .

The current values of the correction factors $\Delta \varphi_{L F(i)}$ and $K c_{(i)}$ used in equation (5) to correct the gauge readings are calculated by the system of equations (10)

$$
\left.\begin{array}{c}
T x 1=K_{L F(1)}(h, j t x) \cdot\left[\varphi_{L F(1)}+\Delta \varphi_{L F(i-1)}+K c_{(i-1)} \cdot \varphi_{L F(1)}\right]+B_{L F(1)}(h, j t x) \\
T 1=K_{L F(1)}(h, j t x) \cdot\left[\varphi_{L F(1)}+\Delta \varphi_{L F(i)}+K c_{(i)} \cdot \varphi_{L F(1)}\right]+B_{L F(1)}(h, j t x) \\
T x 2=K_{L F(2)}(h, j t x) \cdot\left[\varphi_{L F(2)}+\Delta \varphi_{L F(i-1)}+K c_{(i-1)} \cdot \varphi_{L F(2)}\right]+B_{L F(2)}(h, j t x) \\
T 2=K_{L F(2)}(h, j t x) \cdot\left[\varphi_{L F(2)}+\Delta \varphi_{L F(i)}+K c_{(i)} \cdot \varphi_{L F(2)}\right]+B_{L F(2)}(h, j t x)
\end{array}\right\},
$$

where $\Delta \varphi_{L F(i-1)}$ and $K c_{(i-1)}$ are the coefficients used in the previous calibration; $T x 1$ and $T \times 2$ are the thickness gauge readings with the previous correction factor values; $T 1$ and $T 2$ are the values of the thickness of sample pipes.

\section{Conclusion}

The experiments showed that the dual-frequency eddy-current method used to measure the wall thickness of the electrically conductive pipes according to the described algorithms reduces the impact of the gap between the pipe and the eddy-current transducer, and that of the changes in the pipe electrical conductivity on the measurement results.

\section{References}

[1] GOST 23786-79. Drill pipes made of aluminum alloys. Technical conditions (Publishing House of Standards, Moscow, 1990)

[2] E.V. Yakimov, A.E. Goldstein, V.F. Bulgakov, Yu.V. Alkhimov, V.Yu. Belyankov, Test. Diagn. (11), 14 (2014) DOI: 10.14489/td.2014.011.pp.014-018

[3] Z.-G. Zhang, H.-X. Feng, W.-H. Zhao, MATEC Web of Conferencesb 40, 04001 (2016) doi : 10.1051/matecconf/20164004001

[4] A.Y. Petrova, O.N. Chaikovskaya, I.V. Plotnikova, Tech. Phys. J. 60, 592 (2015) doi: $10.1134 / \mathrm{S} 1063784215040222$

[5] A.A. Bespalko, L.V. Yavorovich, P.I. Fedotov, Russ. J. Nondestr. Test. 47, 680 (2011) doi: 10.1134/S1061830911100068

[6] O.V. Galtseva, S.V. Bordunov, N.M. Natalinova, S.V. Mazikov, IOP Conf. Ser.: Mater. Sci. Eng. 132, 012003 (2016) doi: 10.1088/1757-899X/132/1/012003

[7] A.P. Surzhikov, T.S. Frangulyan, S.A. Ghyngazov, N.N. Koval, Nucl. Instrum. Methods Phys. Res., Sect. B 267, 1072 (2009) doi: 10.1016/j.nimb.2009.01.144

[8] R. Kodermyatov, M. Ivanov, M. Yuzhakov, V. Kuznetsov, M. Yuzhakova,

E. Timofeeva, MATEC Web of Conferences 48, 05004 (2016) doi: $10.1051 /$ confmatec/20164805004

[9] A. Gavrilin, B. Moyzes, O. Zharkevich, Journal of Vibroengineering 17, 3495 (2015)

[10] O.V. Galtseva, S.V. Bordunov, S.N. Torgaev, IOP Conf. Ser.: Mater. Sci. Eng. 110, 012094 (2016) DOI: 10.1088/1757-899X/110/1/012094 